\title{
Detecting Personal Life Events from Twitter by Multi-Task LSTM
}

\author{
An-Zi Yen \\ Department of Computer Science and \\ Information Engineering \\ National Taiwan University \\ Taipei, 10617 Taiwan \\ azyen@nlg.csie.ntu.edu.tw
}

\author{
Hen-Hsen Huang \\ Department of Computer Science and \\ Information Engineering \\ National Taiwan University \\ Taipei, 10617 Taiwan \\ hhhuang@nlg.csie.ntu.edu.tw
}

\author{
Hsin-Hsi Chen \\ Department of Computer Science and \\ Information Engineering \\ National Taiwan University, Taiwan \\ MOST Joint Research Center for AI \\ Technology and All Vista Healthcare \\ hhchen@ntu.edu.tw
}

\begin{abstract}
People are used to log their life on the social media platform. Life event can be expressed explicitly or implicitly in a text description. However, a description does not always contain life events related to a specific individual. To tell if there exist any life events and further know their categories is indispensable for event retrieval. This paper explores various LSTM models to detect and classify life events in tweets. Experiments show that the proposed Multi-Task LSTM model with attention achieves the best performance.
\end{abstract}

\section{KEYWORDS}

Lifelogging, personal event detection, social media

\section{INTRODUCTION}

Nowadays people are used to log their life in social media such as Twitter, Facebook, and Instagram. The rich repository personal information is useful for memory recall of individuals. From the post "Eating hamburger on Sunday night", we know what an individual did on Sunday. Extraction of lifelogs in social media helps retrieve the relevant life event of an individual.

Several researches have been done for personal life event detection from social media $[1,2,3,4,6]$. However, most of them focus on detecting the major life events such as Marriage, Job promotions, Exam and Graduation. It cannot support the application of retrieving general life events. Different from the previous work, we aim at detecting whether there exists a life event in tweet, and recognizing its category.

To detect if an individual event occurs from a concise and implicit description is the main issue in this task. For instance, a user may post "iPhone X!" only. The short description might represent the user

\section{ACM Reference format:}

A.Z. Yen, H.H. Huang, and H.H. Chen. 2018. Detecting Personal Life Events from Twitter by Multi-Task LSTM. In The 2018 Web Conference Companion (WWW 2018), April 23-27, 2018, Lyon, France, IW3C2, 2 pages. DOI: https://doi.org/10.1145/3184558.3186909

This paper is published under the Creative Commons Attribution-NonCommercialNoDerivs 4.0 International (CC BY 4.0) license. Authors reserve their rights to disseminate the work on their personal and corporate Web sites with the appropriate attribution. WWW'18 Companion April 23-27, 2018, Lyon, France.

(c) 2018 IW3C2 (International World Wide Web Conference Committee), published under Creative Commons CC BY 4.0 License.

ACM ISBN 978-1-4503-5640-4/18/04.

DOI: https://doi.org/10.1145/3184558.3186909 bought a new cell phone or the iPhone $\mathrm{X}$ was released. The former denotes a lifelog of the user, while the latter does not.

We define three types of tweets in this paper, namely non-event, explicit event, and implicit event. Non-event means a tweet does not log a personal event (e.g. world event). Explicit event means the tweet contains the exact information about "Who did What to Whom Where When and How". Implicit event means whether the tweet is a lifelog of its author or does not depend on human interpretation. "iPhone X!" is an example.

\section{RELATED WORK}

Previous researches on detecting personal life events from Twitter focus on major life events of individuals. The work [4] retrieves the tweets replied with CONGRATULATIONS or CONDOLENCES speech acts, and proposes a pipeline system to identify life events. The retrieved tweets must be major life events, rather than personal daily life events. The work [3] proposes models to extract personal events and generates timeline for individuals from Twitter. Most life events extracted by the proposed models are "graduation", "begin working", etc.

The work [1] classifies 11 major life events by using activity and attention features to train a classifier. The work [2] transforms the representation of posts as syntactic and semantic graphs and identifies the life event in tweets such as "Getting Married", "Having Children", "Death of a Parent", and so on. The work [6] tells whether people participate in events and identifies when the events happened.

In this paper, we focus on personal lifelogging and propose method to detect its existence from Twitter. In addition, we identify the categories of the lifelogs. To the best of our knowledge, this is the first research focusing on detecting lifelog of individuals.

\section{METHODOLOGY}

\subsection{Dataset Construction}

We collect 26,818 Chinese tweets from 18 users who have used Twitter for at least 8 years. For each tweet, annotators should label whether the author describe her/his personal life event in this tweet? If yes, annotators should annotate its type in advance. By using the Cohen's kappa, the agreement value is 0.3165 . 
Table 1: Experimental Results

\begin{tabular}{|c|c|c|c|c|c|c|}
\hline Model & Feature & Type of Tweet (acc) & Categories of Event (F1) & Non & Explicit & Implicit \\
\hline LSTM & word & $64.80 \%$ & $28.70 \%$ & $94.12 \%$ & $40.92 \%$ & $0.91 \%$ \\
\hline LSTM + attention & word & $64.94 \%$ & $28.72 \%$ & $88.14 \%$ & $53.72 \%$ & $0 \%$ \\
\hline LSTM & word + POS & $64.44 \%$ & $28.47 \%$ & $86.03 \%$ & $56.02 \%$ & $0 \%$ \\
\hline LSTM + attention & word + POS & $65.27 \%$ & $28.89 \%$ & $88.42 \%$ & $54.35 \%$ & $0 \%$ \\
\hline MTL- LSTM & word & $64.10 \%$ & $30.98 \%{ }^{\star}$ & $78.39 \%$ & $69.10 \%$ & $1.83 \%$ \\
\hline MTL-LSTM + attention & word & $66.34 \%^{\star}$ & $32.34 \%{ }^{\star}$ & $88.35 \%$ & $56.85 \%$ & $2.61 \%$ \\
\hline MTL-LSTM & word + POS & $65.69 \%$ & $32.98 \% \star$ & $87.15 \%$ & $55.67 \%$ & $4.95 \%$ \\
\hline MTL-LSTM + attention & word + POS & $66.54 \% \star$ & $32.53 \% \star$ & $87.54 \%$ & $57.69 \%$ & $5.35 \%$ \\
\hline
\end{tabular}

We further define 12 categories of life events from the annotation result, including perception, presence, motion, activity, status, health related, commerce, receive and give, social, time related, and other. A tweet may belong to more than one category. We use None to denote there does not exist any events in tweet. For example, "Go shopping with my friends, and Buy a new dress." is an explicit event type of tweet, and the categories of life events in this tweet are activity, commerce, and social.

\subsection{Detection of Personal Life Events}

We propose the following two approaches to detect personal life events from Twitter.

LSTM: For modeling context dependency, the pipelined system adopts LSTM neural networks. The system first identifies the type of a tweet, and then determines the categories of life events based on the result of the first step.

Multi-Task LSTM: The goal of multi-task learning (MTL) is to improve the performance of the main task by jointly training on the auxiliary task [5]. In this paper, the main task is identifying the types of tweets and the auxiliary task is classifying the categories of life events.

Besides the features of vectors of word sequences, we concatenate the features of the parts-of-speech of input words tagged by Stanford POS tagger, and use an attention mechanism for enforcing the LSTM model to attend to the important part of a sentence. Figure 1 shows the architecture of the Multi-Tasking LSTM model.

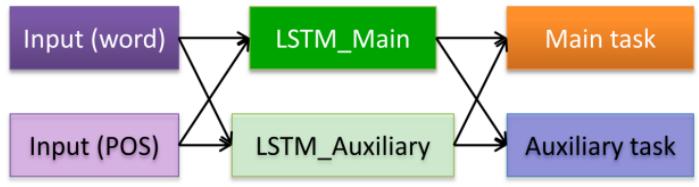

Figure 1: The architecture of the Multi-Tasking LSTM model.

\section{EXPERIMENTS AND EVALUATION}

For the dataset consisting of 26,818 tweets, we remove the tweets which contain photos or only reply to other users. We measure the type of tweet classification by accuracy, and measure the life event classification by F1 score. We use stratified random sampling to split the remaining tweets into training, validation, and test splits. The accuracy is $56.31 \%$ for a classifier that always predicts the majority class (non-event). The top five high frequent categories are perception, motion, activity, presence, and receive and give.

Table 1 shows the performance of our proposed models and the correct ratios in each type predicted by our models. The star $(\star)$ denotes the results are significant with $\mathrm{p}<0.001$ using the McNemar's test comparing with the best LSTM pipelined model.

Comparing the results of the LSTM and the Multi-Task LSTM models, jointly training two models improves the performance of both the type of tweet classification and the life event classification. From the life event classification results, the LSTM model only predicts the high frequent categories. In contrast, the Multi-Task LSTM model can identify more explicit and implicit events. However, the number of implicit events being identified is still small. The reason might be that the content in the implicit event type of tweets are often only food or location. For example, "The Kaiseki level Gyudon" represents the author was eating Gyudon. Therefore, introducing features of named entities or finding other features related to implicit event type is the direction of future work.

\section{CONCLUSIONS}

This paper proposes a Multi-Task LSTM model with attention to identify personal lifelogs from Twitter. Experimental results show that our model can identify non-event and explicit types effectively. The performance of identifying implicit type of events still has room for improvement. In the future, we will introduce more features to classify the type of tweet and the categories of life event.

\section{ACKNOWLEDGMENTS}

This research was partially supported by Ministry of Science and Technology, Taiwan, under grants MOST-105-2221-E-002-154MY3, 106-2923-E-002-012-MY3 and 107-2634-F-002-011-.

\section{REFERENCES}

[1] S. Choudhury and H. Alani. 2015. Detecting Presence of Personal Events in Twitter Streams. In Proceedings of International Conference on Social Informatics, 157-166.

[2] T. Dickinson, M. Fernandez, L. A. Thomas, P. Mulholland, P. Briggs, and H. Alani. 2016. Identifying Important Life Events from Twitter Using Semantic and Syntactic Patterns. In Proceedings of the 15th International Conference WWW/Internet, 143-150.

[3] J. Li and C. Cardie. 2014. Timeline Generation: Tracking Individuals on Twitter. In Proceedings of the 23rd International Conference on World Wide Web, 643-652.

[4] J. Li, A. Ritter, C. Cardie, and E. Hovy. 2014. Major Life Event Extraction from Twitter based on Congratulations/Condolences Speech Acts. In Proceedings of the 2014 Conference on Empirical Methods in Natural Language Processing (EMNLP), 1997-2007.

[5] A. Søgaard and Y. Goldberg. 2016. Deep Multi-task Learning with Low Level Tasks Supervised at Lower Layers. In Proceedings of the 54th Annual Meeting of the Association for Computational Linguistics, 231-235.

[6] K. C. Sanagavarapu, A. Vempala, and E. Blanco. 2017. Determining Whether and When People Participate in the Events They Tweet About. In Proceedings of the 55th Annual Meeting of the Association for Computational Linguistics, 641-646. 